\title{
Proteins pure and applied
}

\section{Protein purification, scaled-up production and analysis.}

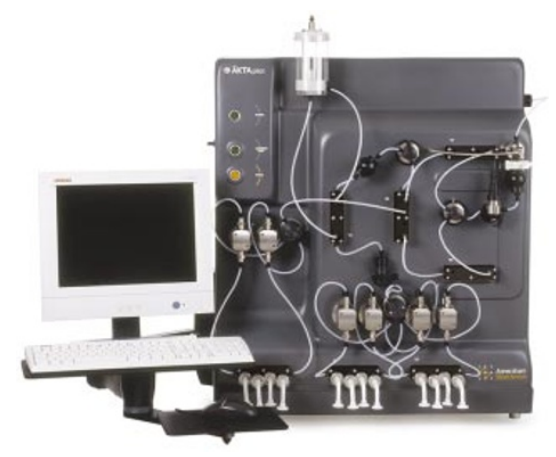

Protein aplenty: AKTApilot for scale-up.

AKTApilot

Amersham Biosciences

www.amershambiosciences.com

The missing link in protein purification

AKTApilot is a bench-top separations system designed to link bench-scale process development with large-scale protein manufacturing. The system offers a range of flow rates, from 4 to $800 \mathrm{ml}$ per min, and allows for multiple wavelength detection as well as $\mathrm{pH}$ and conductivity detection. Eight outlet valves handle fractionation. The system can accommodate an optional Frac-950 and an additional monitor for special detection requirements. It is suitable for scientists who need to perform process development and small-scale production on the same unit.

\section{MessageSensor}

Ambion www.ambion.com

Sensitive RT

The MessageSensor reverse transcriptase (RT) kit contains an $\mathrm{RNase} \mathrm{H}^{+} \mathrm{RT}$ which, according to Ambion, results in greater sensitivity in onestep qRT-PCR experiments than RNase $\mathrm{H}^{-}$ RTs. It will report mRNA abundance over a $10^{6}$-fold range of input RNA. Transcripts can be quantified from as little as $500 \mathrm{fg}$ of total RNA - 1/20th of a single cell. The RT step is completed in 15 minutes. The kit is compatible with one-step, real-time detection methods using TaqMan probes and SYBR Green.

\section{Cryojet HT}

Oxford Instruments (Superconductivity) www.oxford-instruments.com

Broad range of temperatures for $X$-ray crystallography

Crystallographers can investigate molecular structures across a wide range of temperatures (90-490 K) with the Cryojet HT nitrogen jet. The device contains no moving parts: temperature and gas flow settings are adjusted using an electronic controller unit. ObjectBench software enables users to control system settings remotely via computer and perform long-term system monitoring. The storage dewar is at atmospheric pressure, permitting liquid nitrogen refills during use. Temperature remains stable to $\pm 0.1 \mathrm{~K}$, and the system cools down to base temperature rapidly.

\section{Mini-UniPrep}

Whatman Three tools in one

The Whatman Mini-UniPrep is a syringeless filter for removal of particulate from small sample volumes. It combines a syringe, filter and vial in one unit. Proteins can be removed from plasma, serum, whole blood and other biological fluids using the protein precipitation protocol. The method uses acetonitrile precipitation and filtration by compression to remove the protein and, according to Whatman, is three times faster than conventional spin clarification methods and removes over $99 \%$ of protein from plasma samples. It is suitable as an alternative method of removing unwanted protein prior to HPLC/MS analysis in analytical characterization in drug research.

\section{Protein extraction/labelling}

BD Biosciences Clontech www.clontech.com One-step extraction of total cellular protein

The BD Clontech protein extraction and labelling kit provides a gentle, non-denaturing method for preparing a total protein extract of a biological sample. The extraction protocol is fully compatible with a variety of downstream analyses, including two-dimensional polyacrylamide gel electrophoresis, mass spectrometry and immunoassays such as western blots. The kit is also suitable for preparing samples to be used with BD Clontech's Ab microarray, and can be optimized for any mammalian tissue or cell type.

\section{ProteoProfile}

Sigma-Aldrich In-gel N-deglycosylation kit

The ProteoProfile kit is provides a convenient and reproducible method for $N$-deglycosylation and tryptic digestion of protein samples from $1 \mathrm{D}$ or $2 \mathrm{D}$ polyacrylamide gel slices for subsequent mass spectrometry (MS) or highperformance liquid chromatography analysis. Proteomics-grade PNGase F is included to specifically cleave $N$-linked carbohydrates while leaving the peptide structure intact. Proteomics-grade trypsin improves digestion and simplifies MS analysis. The kit works with gel slices or plugs and is suitable for Coomassie Blue and colloidal Coomassie stained gels. It can also be used with gels that have been silver stained and then destained with the company's ProtetoSilver Plus silver staining kit.

\section{Pholipidec}

Advanced Separation Technologies www.astecusa.com Column for separation of key phospholipids

Pholipidec is a stationary phase that can quantify seven key phospholipids present in soy lecithin preparations. It is tailored to account for other contaminants that may be present. The column is suitable for separation of phosphatidylethanolamine, phosphatidylinositol, phosphatidyl serine, phosphatidylcholine (lecithin), phosphatidic acid, lysophosphatidylcholine and lysophosphatidylethanolamine. Column size is $250 \times 4.6 \mathrm{~mm}$, including the integral guard column.

These notes are compiled in the Nature office from information provided by the manufacturers. 